CORRECTION

https://doi.org/10.1038/s41586-018-0814-7

\title{
Author Correction: PAK signalling drives acquired drug resistance to MAPK inhibitors in BRAF-mutant melanomas
}

Hezhe Lu, Shujing Liu, Gao Zhang, Bin Wu, Yueyao Zhu, Dennie T. Frederick, Yi Hu, Wenqun Zhong, Sergio Randell, Norah Sadek, Wei Zhang, Gang Chen, Chaoran Cheng, Jingwen Zeng, Lawrence W. Wu, Jie Zhang, Xiaoming Liu, Wei Xu, Clemens Krepler, Katrin Sproesser, Min Xiao, Benchun Miao, Jianglan Liu, Claire D. Song, Jephrey Y. Liu, Giorgos C. Karakousis, Lynn M. Schuchter, Yiling Lu, Gordon Mills, Yusheng Cong, Jonathan Chernoff, Jun Guo, Genevieve M. Boland, Ryan J. Sullivan, Zhi Wei, Jeffrey Field, Ravi K. Amaravadi, Keith T. Flaherty, Meenhard Herlyn, Xiaowei Xu \& Wei Guo

Correction to: Nature https://doi.org/10.1038/nature24040, published online 27 September 2017.

In this Letter, two relevant references were omitted. The first ${ }^{1}$ shows that exogenous expression of the dominant-negative form of PAK1 sensitizes $B R A F$-mutated, previously untreated melanoma cells to BRAF inhibition (BRAFi) or MEK inhibition (MEKi). This reference, along with several others, was removed from our original submission owing to length limitations. The second ${ }^{2}$ was published during the later stages of the resubmission of our Letter. It shows that inhibition of PAK1 causes re-sensitization to BRAFi of previously untreated cells that ectopically express activated Rac1. Ectopic expression of constitutively active PAK1 increased the tolerance of cells to BRAFi or MEKi. These studies used treatment-naive cells and did not address the issue of acquired drug resistance (that is, cells that showed an initial response but gained resistance over the course of treatment with BRAFi or combined BRAFi and MEKi), which is the central topic of our Letter. Nevertheless, the data support the general notion that PAK1 is involved in regulating resistance to BRAFi and MEKi. We regret not citing these two papers to highlight their relevance and the distinctions between their findings and those of our Letter. The original Letter has not been corrected.

1. Singhal, R. \& Kandel, E. S. The response to PAK1 inhibitor IPA3 distinguishes between cancer cells with mutations in BRAF and Ras oncogenes. Oncotarget 3, 700-708 (2012)

2. Babagana, M. et al. P21-activated kinase 1 regulates resistance to BRAF inhibition in human cancer cells. Mol. Carcinog. 56, 1515-1525 (2017). 